\title{
DYNAMICAL MASS GENERATION IN QUANTUM FIELD THEORY : SOME METHODS WITH APPLICATION TO THE GROSS-NEVEU MODEL AND YANG-MILLS THEORY
}

\author{
DAVID DUDAL A KAREL VAN ACOLEYEN \\ and HENRI VERSCHELDE \\ Ghent University \\ Department of Mathematical Physics and Astronomy \\ Krijgslaan 281-S9 \\ B-9000 GENT, BELGIUM
}

\begin{abstract}
We introduce some techniques to investigate dynamical mass generation. The Gross-Neveu model [a] (GN) is used as a toy model, because the GN mass gap is exactly known 2], making it possible to check reliability of the various methods. Very accurate results are obtained. Also application to $\mathrm{SU}(N)$ Yang-Mills (YM) is discussed.
\end{abstract}

\section{Introduction}

Recently, there was growing evidence that the YM-vacuum favours a condensate of mass dimension 2 [3, 四]. A reasonable candidate is

$$
\Delta=\frac{1}{2}(V T)^{-1}\left\langle\min _{U} \int d^{4} x\left(A_{\mu}^{U}\right)^{2}\right\rangle
$$

where $U$ labels an arbitrary gauge transformation. (11) is a gauge invariant operator and so has some physical meaning. It reduces to the local composite operator (LCO) $\frac{1}{2}\left\langle A_{\mu}^{2}\right\rangle$ in the Landau gauge. The Landau gauge is in a way the most natural one to perform calculations. With another gauge fixing, (11) is no longer local. More precisely, thinking of Abelian projection and imposing the Maximally Abelian Gauge (MAG), we should consider the gauge invariant operator $\Delta=\frac{1}{2}(V T)^{-1}\left\langle\min _{U} \int d^{4} x\left(A_{\mu}^{a} A^{\mu a}\right)^{U}\right\rangle$ where

\footnotetext{
‡ DAVID.DUDAL@RUG.AC.BE

$\S$ RESEARCH ASSISTANT OF THE FUND FOR SCIENTIFIC RESEARCHFLANDERS (BELGIUM)
} 
the index $a$ runs only over the off-diagonal gluons. In MAG, this simplifies to the LCO $\frac{1}{2}\left\langle A_{\mu}^{a} A^{\mu a}\right\rangle$.

We mentioned MAG, because interesting attempts have been undertaken by Kondo e.a. [5] and recently Freire [6] to construct low energy effective theories starting from the full YM Lagrangian. Their efforts rest mainly on the principle of Abelian dominance [7, 8], which can be understood by means of massive off-diagonal gluons and the Appelquist-Carazzone decoupling theorem [9]. MAG requires a 4-ghost interaction to preserve renormalizibility [10], and this non-trivial ghost interaction was used in [11, 12] to produce an effective charged gluon mass. Our analysis learned that, in contrast to their results, the ghost condensate alone resulted in a tachyonic mass. We guess that a combination" of the off-diagonal ghost and gluon condensate might provide us with a real mass.

For the sake of simplicity, we present the different approaches using the GN-model. Available results on YM in the Landau gauge will be quoted too.

\section{Renormalizable effective potential for LCO}

\subsection{GROSS-NEVEU}

We start from the manifestly $\mathrm{U}(N)$-invariant GN Lagrangian in $2-\varepsilon$ dimensional Euclidean spacetime with a source $J$ coupled to the LCO $\bar{\psi} \psi$

$$
\mathcal{L}=\bar{\psi}(\not \partial+J) \psi-\frac{1}{2} g^{2} \mu^{\varepsilon}(\bar{\psi} \psi)^{2}+\mathcal{L}_{\text {counter }}
$$

This is an asymptotically free theory with a chiral $\gamma_{5}$ symmetry if $J=0$, preventing a perturbative non-zero value for $\langle\bar{\psi} \psi\rangle$ and the fermion mass. When $J \neq 0$, new logarithmic infinities $\propto J$ (multiplicative mass renormalization) and $\propto J^{2}$ (vacuum energy divergences) appear. In order to remove the latter, a new coupling $\zeta$ must be incorporated into (2), and we get

$$
\mathcal{L}=\bar{\psi}(\not \partial+J) \psi-\frac{1}{2} g^{2} \mu^{\varepsilon}(\bar{\psi} \psi)^{2}-\frac{1}{2} \mu^{-\varepsilon} \zeta J^{2}+\mathcal{L}_{\text {counter }}
$$

where $\mathcal{L}_{\text {counter }}$ contains all counterterm information.

$$
\mathcal{L}_{\text {counter }}=\delta Z \bar{\psi}(\not \partial) \psi+\delta Z_{2} J \bar{\psi} \psi-\frac{1}{2} \delta Z_{g} g^{2} \mu^{\varepsilon}(\bar{\psi} \psi)^{2}-\frac{1}{2} \mu^{-\varepsilon} \delta \zeta J^{2}
$$

\footnotetext{
${ }^{1}$ Some more formal results on this topic were obtained in 13 .
} 
Defining the bare quantities as

$$
\begin{aligned}
\psi_{o} & =\sqrt{Z} \psi \\
J_{o} & =\frac{Z_{2}}{Z} J \\
g_{o}^{2} & =\frac{Z_{g}}{Z^{2}} g^{2} \\
\zeta_{o} J_{o}^{2} & =\mu^{-\varepsilon}(\zeta+\delta \zeta) J^{2}
\end{aligned}
$$

the finite, generating energy functional $E(J)$ satisfies a homogeneous renormalization group equation (RGE)

$$
\left(\mu \frac{\partial}{\partial \mu}+\beta\left(g^{2}\right) \frac{\partial}{\partial g^{2}}-\gamma_{2}\left(g^{2}\right) \int d^{2} x J \frac{\delta}{\delta J}+\eta\left(g^{2}, \zeta\right) \frac{\partial}{\partial \zeta}\right) E=0
$$

with

$$
\begin{aligned}
\beta\left(g^{2}\right) & =\left.\mu \frac{\partial}{\partial \mu} g^{2}\right|_{g_{o}, \varepsilon} \\
\gamma_{2}\left(g^{2}\right) & =\left.\mu \frac{\partial}{\partial \mu} \ln \frac{Z_{2}}{Z}\right|_{g_{o}, \varepsilon} \\
\eta\left(g^{2}, \zeta\right) & =\left.\mu \frac{\partial}{\partial \mu} \zeta\right|_{g_{o}, \varepsilon, J_{o}, \zeta_{o}}
\end{aligned}
$$

The above reasoning to treat a $\mathrm{LCO}$ seems to have 2 problems : First, $\zeta$ is at this stage still arbitrary, so we have a problem of uniqueness. Putting $\zeta=0$ is a bad choice, it leads to a non-homogeneous RGE for $E$ and a non-linear RGE for the effective action $\Gamma$. Secondly, the $J^{2}$ term spoils a simple energy interpretation. These are reflections of the criticism uttered by Banks and Raby on the use of LCO 14. Both problems can be solved by choosing $\zeta$ such a function of $g^{2}$, so that if $g^{2}$ runs according to $\beta, \zeta$ will run properly according to $\eta$ [15]. Indeed, because of (9) and (10), we have

$$
\mu \frac{\partial}{\partial \mu} \zeta=\eta=2 \gamma_{2} \zeta+\delta
$$

where

$$
\delta=\varepsilon \delta \zeta-\mu \frac{\partial}{\partial \mu} \delta \zeta+2 \gamma_{2} \delta \zeta
$$

A solution of (13) is $\zeta=\zeta\left(g^{2}\right)$ where $\zeta\left(g^{2}\right)$ is a particular solution of

$$
\beta\left(g^{2}\right) \frac{d}{d g^{2}} \zeta\left(g^{2}\right)=2 \gamma_{2}\left(g^{2}\right) \zeta\left(g^{2}\right)+\delta\left(g^{2}\right)
$$

The integration constant has been put to zero, in order to avoid an independent coupling constant and to have multiplicatively renormalizable vacuum 
divergences $\left(\zeta+\delta \zeta=Z_{\zeta} \zeta\right)$. We will solve (15) by a Laurent expansion $\zeta=\frac{z_{-1}}{g^{2}}+z_{0}+z_{1} g^{2}+\ldots$ Notice that $n$-loop results require $(n+1)$-loop knowledge of $\beta, \gamma_{2}$ and $\delta$. The generating functional $E$ now fulfills

$$
\left(\mu \frac{\partial}{\partial \mu}+\beta\left(g^{2}\right) \frac{\partial}{\partial g^{2}}-\gamma_{2}\left(g^{2}\right) \int d^{2} x J \frac{\delta}{\delta J}\right) E=0
$$

We conclude that the LCO $\Delta=Z_{2} \bar{\psi} \psi-Z_{\zeta} \zeta J$ has a finite and multiplicatively renormalizable $\operatorname{VEV}\langle\Delta\rangle=\frac{\delta W}{\delta J}$. The effective action $\Gamma(\Delta)$, obeys the following RGE

$$
\left(\mu \frac{\partial}{\partial \mu}+\beta\left(g^{2}\right) \frac{\partial}{\partial g^{2}}+\gamma_{2}\left(g^{2}\right) \int d^{2} x \Delta \frac{\delta}{\delta \Delta}\right) \Gamma(\Delta)=0
$$

Introducing unity via the Hubbard-Stratonovich transformation

$$
1=\int[d \sigma] \exp -\frac{1}{2 Z_{\zeta} \zeta} \int d^{2-\varepsilon} x\left[\frac{\sigma}{g}+\mu^{\frac{\varepsilon}{2}} Z_{2} \bar{\psi} \psi-\mu^{\frac{-\varepsilon}{2}} Z_{\zeta} \zeta J\right]^{2}
$$

we finally arrive at

$$
\exp (-E(J))=\int[d \bar{\psi} d \psi d \sigma] \exp -\int d^{2-\varepsilon} x\left[\mathcal{L}(\sigma, \bar{\psi}, \psi)-\mu^{\frac{-\varepsilon}{2}} \frac{\sigma}{g} J\right]
$$

where

$$
\mathcal{L}=Z \bar{\psi} \not \partial \psi-\frac{1}{2} \mu^{\varepsilon} g^{2}(\bar{\psi} \psi)^{2}\left[Z_{g}-\frac{Z_{2}^{2}}{g^{2} Z_{\zeta} \zeta}\right]+\frac{\sigma^{2}}{2 g^{2} Z_{\zeta} \zeta}+\mu^{\frac{\varepsilon}{2}} g \sigma \bar{\psi} \psi \frac{Z_{2}}{g^{2} Z_{\zeta} \zeta}
$$

$J$ is now a real source, in the sense that it appears linearly for $\sigma$ so that we have a straightforward energy interpretation and $\langle\sigma\rangle=\langle-g \Delta\rangle$. Eq.(20) is a new effective, renormalized Lagrangian for GN, equivalent to the original (2) but encapsulating non-perturbative information. Perturbing around $\sigma=0$, we recover the original perturbation series with its infrared renormalon problems. If we calculate the effective potential for $\sigma \neq 0$, we could perturb around a non-perturbative vacuum free of renormalons.

We won't rederive $V(\sigma)$, the results can be found in the original papers [15]. Out of (20), we immediately see that $\langle\sigma\rangle \neq 0$ gives birth to a fermion mass. After improving the renormalization prescriptions, very accurate results for the effective fermion mass were obtained (see TABLE I). For comparison, we also displayed the $N \rightarrow \infty$ and $1 / N$ results. 
TABLE I. Deviation in terms of percentage for the mass gap with LCO method

\begin{tabular}{cccc}
\hline$N$ & 2-loop mass gap & $N \rightarrow \infty$ mass gap & $1 / N$ mass gap \\
\hline 2 & $41.67 \%$ & $-46.3 \%$ & $-21.9 \%$ \\
3 & $7.13 \%$ & $-32.5 \%$ & $-12.2 \%$ \\
4 & $2.84 \%$ & $-24.2 \%$ & $-7.0 \%$ \\
5 & $1.53 \%$ & $-19.1 \%$ & $-4.5 \%$ \\
6 & $0.97 \%$ & $-15.8 \%$ & $-3.1 \%$ \\
7 & $0.68 \%$ & $-13.5 \%$ & $-2.3 \%$ \\
8 & $0.51 \%$ & $-11.7 \%$ & $-1.8 \%$ \\
9 & $0.40 \%$ & $-10.4 \%$ & $-1.4 \%$ \\
10 & $0.33 \%$ & $-9.3 \%$ & $-1.1 \%$ \\
\hline
\end{tabular}

\subsection{YANG-MILLS WITH LANDAU GAUGE FIXING}

The same approach has been employed to investigate the condensate suggested in (11). There are some subtleties since YM is a gauge theory [16]. The corresponding effective YM-Lagrangian, was found to be

$$
\begin{aligned}
\mathcal{L}\left(\sigma, A_{\mu}\right) & =\frac{1}{4} F_{\mu \nu}^{a} F_{\mu \nu}^{a}+\mathcal{L}_{\text {gauge }+ \text { F.P. }}+\mathcal{L}_{\text {counter }}+\frac{\sigma^{2}}{2 g^{2} Z_{\zeta} \zeta} \\
& +\frac{1}{2} \mu^{\frac{\varepsilon}{2}} g \sigma A_{\mu}^{a} A_{\mu}^{a} \frac{Z_{2}}{g^{2} Z_{\zeta} \zeta}+\frac{1}{8} \mu^{\varepsilon} \frac{Z_{2}^{2}}{Z_{\zeta} \zeta}\left(A_{\mu}^{a} A_{\mu}^{a}\right)^{2}
\end{aligned}
$$

$V(\sigma)$ was computed up to 2-loop order using the $\overline{M S}$ scheme. RG-improved perturbation theory showed that all gluons are massive :

$$
m_{\text {gluon }} \approx 465 \mathrm{MeV} \text { with } \frac{g^{2} N}{16 \pi^{2}} \approx 0.14466
$$

Note that the relevant expansion parameter is relatively small, so perturbation theory can be qualitatively trusted.

\section{Dynamical mass generation by source inversion}

The second tool we discuss, was worked out recently by Van Acoleyen et al. [17].

When the GN model is probed with a source $J$, just as in (2), one can calculate the effective mass $m(J)$ as a function of $J$. Due to the asymptotic 
freedom, this expansion is only valid for large $J$. To recover the original GN model, we must take the limit $J \rightarrow 0$. Doing so, the perturbation series for $m(J)$ blows up and no relevant information can be extracted.

However, it is possible to invert the relation $m(J)$ to $J(m)$. If a sufficiently large solution $m_{\star}$ of $J(m)=0$ exists, we can consider the limit of vanishing source, while the perturbative expansion remains valid.

The solution $m_{\star} \neq 0$ will be renormalization scheme and scale dependent, due to the arbitrary renormalization prescriptions. To remove this freedom, we proceed in the following way. $J$ runs according to its renormalization group equation as

$$
\mu \frac{\partial J}{\partial \mu}=-\gamma_{2}\left(g^{2}\right) J
$$

$J$ is a scheme and scale dependent quantity, with the result that the equation for the mass gap is also scheme and scale dependent. But it is easily checked that $\tilde{J}$, defined by

$$
\tilde{J}=f\left(g^{2}\right) J
$$

where $f\left(g^{2}\right)$ is a solution of

$$
\mu \frac{\partial f}{\partial \mu}=\gamma_{2} f
$$

is scheme and scale independent (SSI).

When we transform $J$ to $\tilde{J}$, the gap equation becomes $\tilde{J}(m)=0$, since $\tilde{J} \propto J$. Because $\tilde{J}$ is SSI, the gap equation is SSI, so $m_{\star}$ will be SSI.

Since we can calculate the perturbative series for $m(J)$ an $\tilde{J}$ only up to a certain order, there will always be a remnant of scheme and scale dependence. By exchanging the expansion parameter $g^{2}(\mu)$ for $\frac{1}{\beta_{0} \ln \frac{\mu^{2}}{\Lambda^{2}}}(\Lambda$ is the scale parameter of the renormalization scheme), it is possible to rewrite $\tilde{J}$ as $\tilde{J}=m \mathcal{J}\left(\frac{m}{\Lambda \overline{M S}}\right)$, where $\mathcal{J}$ is a series in $\frac{1}{\beta_{0} \ln \frac{m^{2}}{\Lambda^{2}}+d}$ with all scheme and scale dependence settled in the parameter $d$.

When we would include all orders in the calculation, $d$ would drop out of the result. At finite order precision, $d$ will be present in the final result for $m_{\star}$. We can fix $d$ by using the principle of minimal sensitivity (PMS) 18 by demanding that the mass gap $m_{\star}$ has minimal dependence on $d\left(\Leftrightarrow \frac{\partial m_{\star}}{\partial d}=0\right)$. At 2 loop order, PMS did give an optimal $d$ and the corresponding mass was close to the exact mass (see TABLE II). 
TABLE II. Deviation in terms of percentage for the mass gap with source inversion method

\begin{tabular}{cccc}
\hline$N$ & 2-loop mass gap & $N \rightarrow \infty$ mass gap & $1 / N$ mass gap \\
\hline 2 & $\pm 20 \%$ & $-46.3 \%$ & $-21.9 \%$ \\
3 & $0.9 \%$ & $-32.5 \%$ & $-12.2 \%$ \\
4 & $-1.0 \%$ & $-24.2 \%$ & $-7.0 \%$ \\
5 & $-1.5 \%$ & $-19.1 \%$ & $-4.5 \%$ \\
6 & $-1.6 \%$ & $-15.8 \%$ & $-3.1 \%$ \\
7 & $-1.6 \%$ & $-13.5 \%$ & $-2.3 \%$ \\
8 & $-1.5 \%$ & $-11.7 \%$ & $-1.8 \%$ \\
9 & $-1.4 \%$ & $-10.4 \%$ & $-1.4 \%$ \\
10 & $-1.3 \%$ & $-9.3 \%$ & $-1.1 \%$ \\
\hline
\end{tabular}

The source inversion method was also tested on the chiral Gross-Neveu model, again with good results 19 .

\section{Summary}

We have dealt with 2 different approaches concerning dynamical mass generation. Each method seems to give (very good) results in case of GN. The almost exact GN results doesn't mean we can generalize immediately to the YM case (in the Landau gauge). Other sources of non-perturbative effects besides infrared renormalons, such as instantons, will contribute to the dynamical mass.

In the future, also MAG YM deserves our attention, first trying to clear the widely accepted Abelian dominance in a somewhat analytical way. Secondly, there might exist a connection between those condensate formations and the Curci-Ferrari Lagrangian [20, 21].

As a general conclusion, we state it is possible to get non-perturbative information on e.g. a dynamical gluon mass in a sector of the YM-vacuum accessible to perturbation theory. 


\section{References}

1. D.J. Gross, A. Neveu, Phys.Rev. D10 (1974) 3235

2. P. Forgács, F. Niedermayer, P. Weisz, Nucl.Phys. B367 (1991) 123

3. F.V. Gubarev, L. Stodolsky, V.I. Zakharov, Phys.Rev.Lett.86 (2001) 2220

4. F.V. Gubarev, V.I. Zakharov, Phys.Lett. B501 (2001) 28

K.G. Chetyrkin, S. Narison, V.I. Zakharov, Nucl.Phys. B550 (1999) 353

5. K.I. Kondo, hep-th/0009152

K.I. Kondo, T. Shinohara, Prog.Theor.Phys.105 (2001) 649

K.I. Kondo, Phys.Rev.D57 (1998) 7467

6. F. Freire, Phys.Lett. B526 (2002) 405

7. T. Suzuki, I. Yotsuyanagi, Phys.Rev. D42 (1990) 4257

H. Hioki, S.Kitahara, S. Kiura, Y. Matsubara, O. Miyamura, S. Ohno, T. Suzuki, Phys.Lett. B272 (1991) 326, erratum ibid.B281 (1992) 416

8. K. Amemiya, H. Suganuma, Phys.Rev. D60 (1999) 114509

K. Amemiya, H. Suganuma, Nucl.Phys.Proc.Suppl. 83 (2000) 419

9. T. Appelquist, J. Carazzone, Phys.Rev. D11 (1975) 2856

10. H. Min, T. Lee, P.Y. Pac, Phys.Rev. D32 (1985) 440

11. K.I. Kondo, T.Shinohara, Phys.Lett. B491 (2000) 263

12. M. Schaden, hep-th/9909011, hep-th/0003030, hep-th/0108034

13. K.I. Kondo, Phys.Lett. B514 (2001) 335

K.I. Kondo, T. Murakami, T. Shinohara, T. Imai, Phys.Rev. D65 (2002) 085034

14. T. Banks, S. Raby, Phys.Rev. D14 (1976) 2182

15. H. Verschelde, Phys.Lett. B351 (1995) 242

H. Verschelde, S. Schelstraete, M. Vanderkelen, Z.Phys. C76 (1997) 161

16. H. Verschelde, K. Knecht, K. Van Acoleyen, M. Vanderkelen, Phys.Lett. B516 (2001) 307

17. K. Van Acoleyen, H. Verschelde, Phys.Rev. D65 (2002) 085006

18. P.M. Stevenson, Phys.Rev. D23 (1981) 2916

19. K. Van Acoleyen, J.A. Gracey, H. Verschelde, hep-th/0202194, to appear in Phys.Rev. D

20. G. Curci, R. Ferrari, Nuovo Cim. A32 (1976) 151

G. Curci, R. Ferrari, Nuovo Cim. A35 (1976) 1, erratum ibid. A47 (1978) 555

G. Curci, R. Ferrari, Nuovo Cim. A35 (1976) 273

21. J.A. Gracey, Phys.Lett. B525 (2002) 89 